\title{
Tools for prediction of xenobiotics interaction with human cytochrome P450
}

\author{
A Dmitriev*, D Filimonov, A Lagunin and V Poroikov
}

Address: Institute of Biomedical Chemistry of RAMS, Pogodinskaya Str. 10, 119121, Moscow, Russia

* Corresponding author

from 4th German Conference on Chemoinformatics

Goslar, Germany. 9-II November 2008

Published: 5 June 2009

Chemistry Central Journal 2009, 3(Suppl I):P8 doi:I0.1186/I752-I53X-3-SI-P8

This abstract is available from: http://www.journal.chemistrycentral.com/content/3/SI/P8

(C) 2009 Dmitriev et al; licensee BioMed Central Ltd.

Since many exogenous substances, including ecotoxicants, pharmaceuticals, food additives, and others are undergone to metabolic transformations by cytochrome $\mathrm{P} 450$, information about their fate in the human organism is very important. Comparing to the parent compounds, metabolites produced by biotransformation reactions may reveal higher toxicity (e.g., having carcinogenic potency) or may have more potent biological activity (this property is used in case of prodrugs). Induction or inhibition of cytochromes P450, which take part in biotransformation, may lead to decrease or increase of biological effects of ecotoxicants and drugs. Development of software for computer prediction of biotransformation of xenobiotics in the human organism and cytochrome P450 substrate/inhibition/induction activity for drug-like compounds was performed on the basis of PASS technology http://www.ibmc.msk.ru/PASS/. One created software product is designed as a tool for prediction of biotransformation classes (e.g. "N-Glucuronidation", "Acylation", "N-Hydroxylation, Cytochrome P450, CYP3A4" etc.) and substrate/inhibitor/inducer activity (CYP3A4 inducer, CYP2C9 substrate, CYP2C19 inhibitor etc.) of drug-like compounds in the human organism. Training set is used for prediction of biotransformation spectra for new chemical compounds. Created software product predicts about 400 kinds of biotransformation terms with the mean prediction accuracy of about $88 \%$. For validation and testing of program we used the test set created on the basis of information presented in "The Top 100 Drug Interaction 2006 H\&H Publications". The test set contains 260 chemical structures of drugs and data about their substrate/inhibition/induction properties.
Interaction with the CYP2C19, CYP3A4, CYP2D6, CYP2C9, CYP1A2 isoforms of cytochrome $\mathrm{P} 450$ was predicted. Accuracy of prediction is $74 \%$, sensitivity is $69 \%$, specificity is $75 \%$. Another software product is designed as a tool for prediction of C-Hydroxylation sites for drug-like compounds in the human organism. In current version, program is able to predict C-Hydroxylation sites for compounds in the human organism with the mean prediction accuracy of about $90 \%$. If the appropriate training set and biotransformation fragments will be available, the program can be extended to prediction of sites for other biotransformation. Prediction of C-Hydroxylation biotransformation sites is realized by using of RMNA descriptors and metabolite generation procedure. For validation of the program, prediction versus random tests sets was performed. Two test set contains 130 and 260 structures xenobiotics. Accuracy of C-Hydroxylation predictions for two test sets and for various levers of RMNA descriptors is about $70 \%$. Results obtained of C-Hydroxylation predictions and prediction of xenobiotics interaction with the main isoforms of cytochrome P450 look reasonable to use them for solution of practical tasks of toxicology and pharmacology. 